\title{
Thermally Sprayed Wear Resistant Coatings With Nanostructured Hard Phases
}

\author{
Stephan Siegmann, Oliver Brandt, and Michael Dvorak
}

(Submitted 3 November 2001; in revised form 3 March 2002)

\begin{abstract}
The aim of this investigation was the development of a new quality of thermally sprayed coatings with high resistance against wear and corrosion and to evaluate the application potential of nano-sized hard phases in thermally sprayed layers. The newly developed material consists of a highly corrosion-resistant matrix of stainless steel (even without nickel) combined with nano-structured hard phases of vanadium nitrides (VN). On the other hand, matrices consisting of cobalt-chromium $(\mathrm{CoCr})$ with submicron hard phases of tungstencarbides (WC) were investigated with respect to microstructure and wear resistance compared with conventional ones.
\end{abstract}

Keywords comparison, hard chromium, HVOF, nanostructured coatings, thermal spraying, VPS, wear resistance

\section{Introduction}

Nanomaterials are gaining interest due to their potential advantages over the properties of material with the same chemistry but conventional structure. As is known from different publications (c.g., Ref 1-4), the smaller the (hard) phases are, the higher the wear resistance of the eorresponding coatings can be.

For this reason, the following approach for creating a new kind of powder and coating investigations were made.

Two composite materials with different matrices (stainless steel or $\mathrm{CoCr}$ ) and submicron hard phases (VN, WC) were developed and tested using different thermal spraying facilities like vacuum plasma spraying (VPS) and high velocity oxygen fuel spraying (HVOF).

To have a good corrosion resistance, the matrix metals have to be completely alloyed and corrosion resistant thiemselves. In addition, such coatings must be dense and have no open porosity. Finally, the different groups of nano hard phases (type VN, WC, etc.) are responsible for the good wear resistance.

For the first category of powders, consisting of two types of vanadium nitrides (primary nitrides of micrometer size and secondary nitrides of nanometer size) three processing routes were investigate $\mathrm{d}^{[5-7]}$ :

1. Heat treating of the starting powder material under nitrogen atmosphere to promote the formation of nitrides and preserving this stiucture during thermal spraying

2. Formation of the nitrides during reactive thermal spraying of previously unreacted powder

3. Post nitridation of the conventionally sprayed coatings with unreacted powder

Stephan Siegmann, Swiss Federal Laboratories for Materials Testing and Research, Feuerwerkerstrasse 39 CH-3602 Thun, Switzerland; Oliver Brandt, Brandt Engineering and Consulting Feuerwerkerstr. 39 CH-3602 Thun, Switzerland; and Michael Dvorak, DACS Dvorak Advanced Coating Solutions, Feuerwerkerstr 39 CH-3602 Thun, Switzerland. Contact e-mail: stephan.siegmann@empa.ch.
A comparison of different thermal spraying techniques like flame spraying (FS), high velocity flame spraying (HVOF), and plasma spraying (APS, VPS) demonstrated their specific advantages while preserving or creating the desired phase structures. ${ }^{[5-8]}$

The other category of powder material consisted of very fine tungsten carbides ( $\mathrm{fc}=$ fine carbides) embedded in a cobaltchromium matrix (fc-WC-CoCr) and conventional WC-CoCr. The coating formation was done using a conventional Kerosene driven high-pressure HVOF system (Terojet) (Eutectic-Castolin SA, Lausanne, Switzerland), and for comparison, a VPS equipment.

A study of the final coating qualities, including the resistance against abrasive and erosive wear compared with conventionally used coatings, like other thermally sprayed deposits or hard chrome plating, was carried out.

\section{Powder Investigations}

Two different types of spraying materials were investigated: nanostructured vanadium-nitrides and tungsten-carbide cobaltchromium.

\subsection{Nanostructured Vamadium-Nitrides}

Characteristics like high toughness, high corrosion, and simultaneous wear resistance can be achieved by the correct combination of the alloying elements like chromium $(\mathrm{Cr})$, molybdenum (Mo), and nitrogen $(\mathrm{N})$. Based on previous alloying studies with various hot isostatically pressed (HIP-compacted) bulk materials, ${ }^{[9]}$ an optimized iron(Fe)-based alloy consisting of Fe17Cr10Mn6VMo was developed. Vanadium combined with nitrogen and a heat treatment, like the VPS process, will form very fine nitrides that influence the mechanical, tribological, and chemical properties of the resulting coatings. The nitridation process causes also a transformation of the ferritic into an austenitic structure. This material may therefore also be regarded as a hardenable corrosion-resistant austenitic iron-based nano metal matrix composite (MMC).

With regard to the demand of thermal spraying, a spherical powder (Fig. 1) was produced by a gas atomization process. The size of the VN and their size distribution can be adjusted as a 

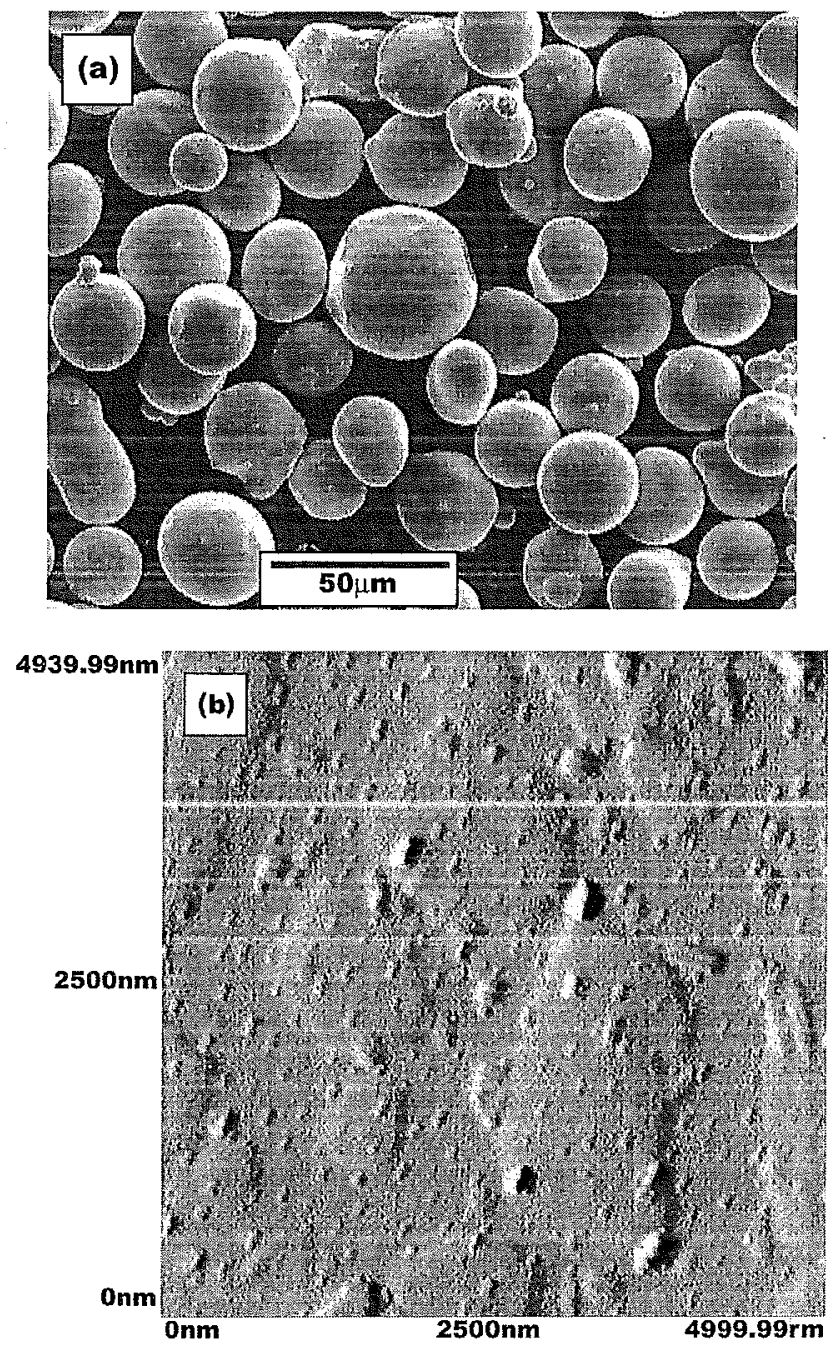

Fig. 1 (a) Typical morphology of the Fe17Cr10Mn6VMo gas atomized powder showing a particle size range $-45+25 \mu \mathrm{m}$ and (b) AFM picture of the nitrided powder showing hard phases sticking out of the softer matrix after polishing

function of the $\mathrm{N}$ content during the formation process. ${ }^{[7]}$ The final powder was sieved into three fractions $(-25+5 \mu \mathrm{m},-45+$ $25 \mu \mathrm{m}$, and $-90+45 \mu \mathrm{m})$. The baseline studies were carried out using the powder having the medium fraction $-45+25 \mu \mathrm{m}$.

2.1.1 Vacuum Plasma Spraying. Using a VPS facility, coatings can be sprayed without any oxidation during coating formation and with excellent coating properties. The coating application was done on a VPS-system (Medicoat AG, Mägenwil, Switzerland) with a MC-60 torch. A wide range of operation conditions was investigated to study the influence of nitridation on the resulting coating properties.

Beside the preceding nitridation of the starting powders, the reactive alloying with $\mathrm{N}$ can be achieved during vacuum plasma spraying by the exposition of the particles to a $\mathrm{N}_{2}$-rich plasma and by creating a $\mathrm{N}_{2}$-rich environment in the VPS chamber. Tests have shown that the most efficient way of $\mathrm{N}_{2}$ alloying during VPS processing was the use of a plasma consisting of an $\mathrm{Ar} / \mathrm{N}_{2}$ mixture (Fig. 2 and 3). ${ }^{[7]}$

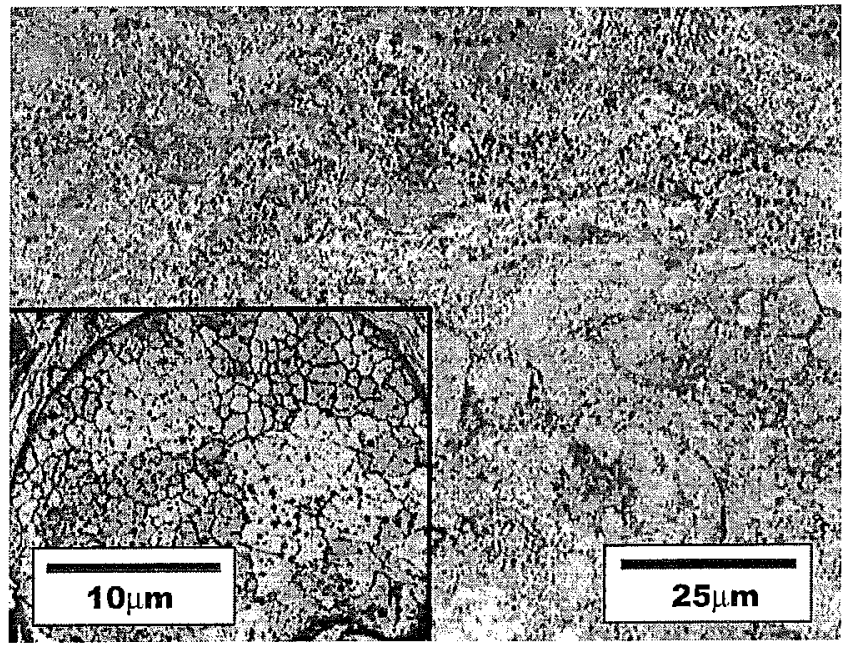

Fig. 2 Optical microstructure of a typical Fe17Cr10Mn6VMo coat ing, showing well-distributed dark primary Vanadium nitrides after chemical etching.

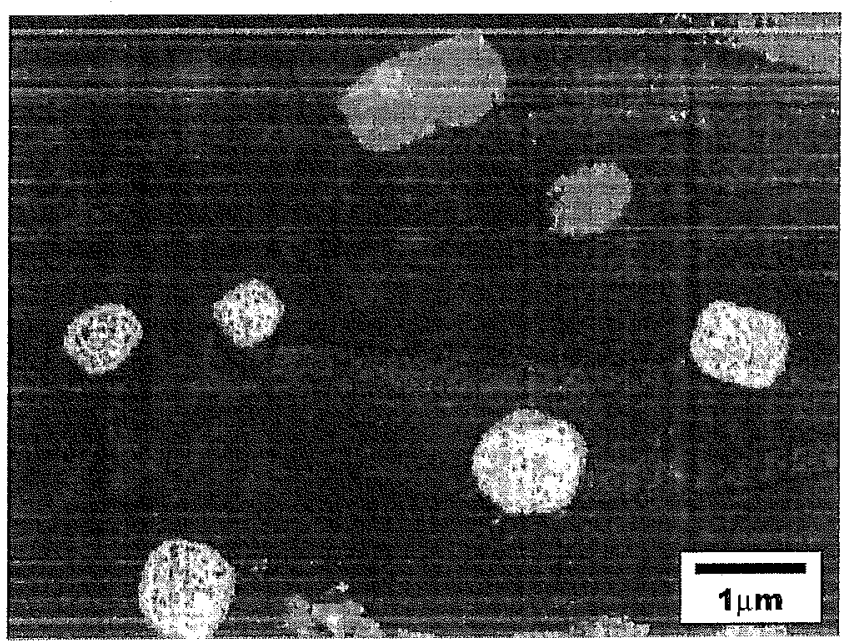

Fig. 3 TEM image showing primary nitrides of micrometer size ${ }^{[8]}$

To clarify the effect of $\mathrm{N}_{2}$-alloying as a function of spray powder particle size, Fe17Cr10Mn6VMo powders with three different size ranges were sprayed.

A decreasing $\mathrm{Ar} / \mathrm{N}_{2}$-mixture yielded an increasing electrical input power (EIP) and an increase in $\mathrm{N}_{2}$ content of the final coating up to $3 \mathrm{wt} . \%$. When spraying is performed with the powder fraction of $-90+45 \mu \mathrm{m}$, the increase in $\mathrm{N}_{2}$-content in the coating with increasing EIP is marginal compared to that during spraying with finer powders. When spraying is performed with an EIP of $45 \mathrm{~kW}$, the resulting $\mathrm{N}_{2}$ content of the fine fraction $-25+5 \mu \mathrm{m}$ was close to the maximum value reached in this study. Changing the EIP from 45 to $47 \mathrm{~kW}$ yielded an increase of the $\mathrm{N}_{2}$ content in the coating from $1 \mathrm{wt} . \%$ to more than $3 \mathrm{wt} . \%$.

The formation of the additional secondary nitrides of nanometer size can be detected only by transmission electron microscopy (TEM) or atomic force microscopy (AFM). TEM and electron energy loss spectroscopy (EELS) investigations showed the 


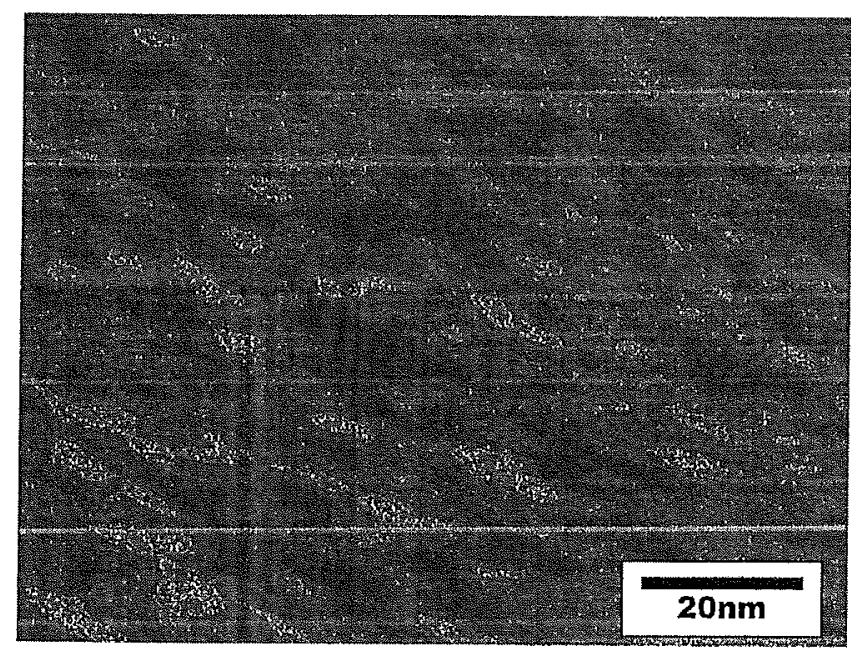

Fig. 4 TEM image showing secondary nitrides of nanometer size $\mathrm{e}^{[8]}$

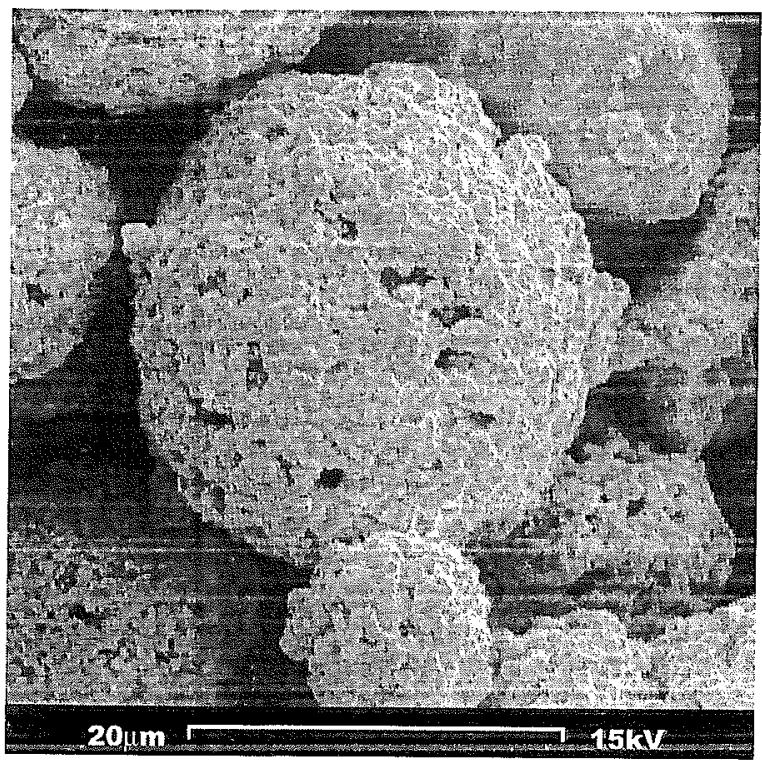

Fig. 5 SEM picture of the (fc) WC-CoCr particles

existence of elongated secondary VN $20-30 \mathrm{~nm}$ in length and 5 $\mathrm{nm}$ in width (Fig. 4).

\subsection{Tungsten-Carbide Cobalt-Chromium}

The WC-CoCr 86-10-4 powders used for this study were produced by WOKA (Barchfeld, Germany) and were in a conventional and a fine (i.e., narrow) distribution. ${ }^{[10]}$ The expected mean grain size for the fine WC powders ( $f \mathrm{c}$ ) was $0.8 \mu \mathrm{m}$. Also, the carbide distribution was expected to be more homogenous by using finer raw materials. As a matrix raw material, extra fine Co and $\mathrm{Cr}$ was used to ensure complete alloying of the metals during the sintering. The depth of the diffusion depended on the sintering time and the sintering temperature. To avoid any formation of $\eta$-carbide phases, the WC were added to the process with a strictly controlled carbon (C) content of 6.0-6.2 wt.\%.
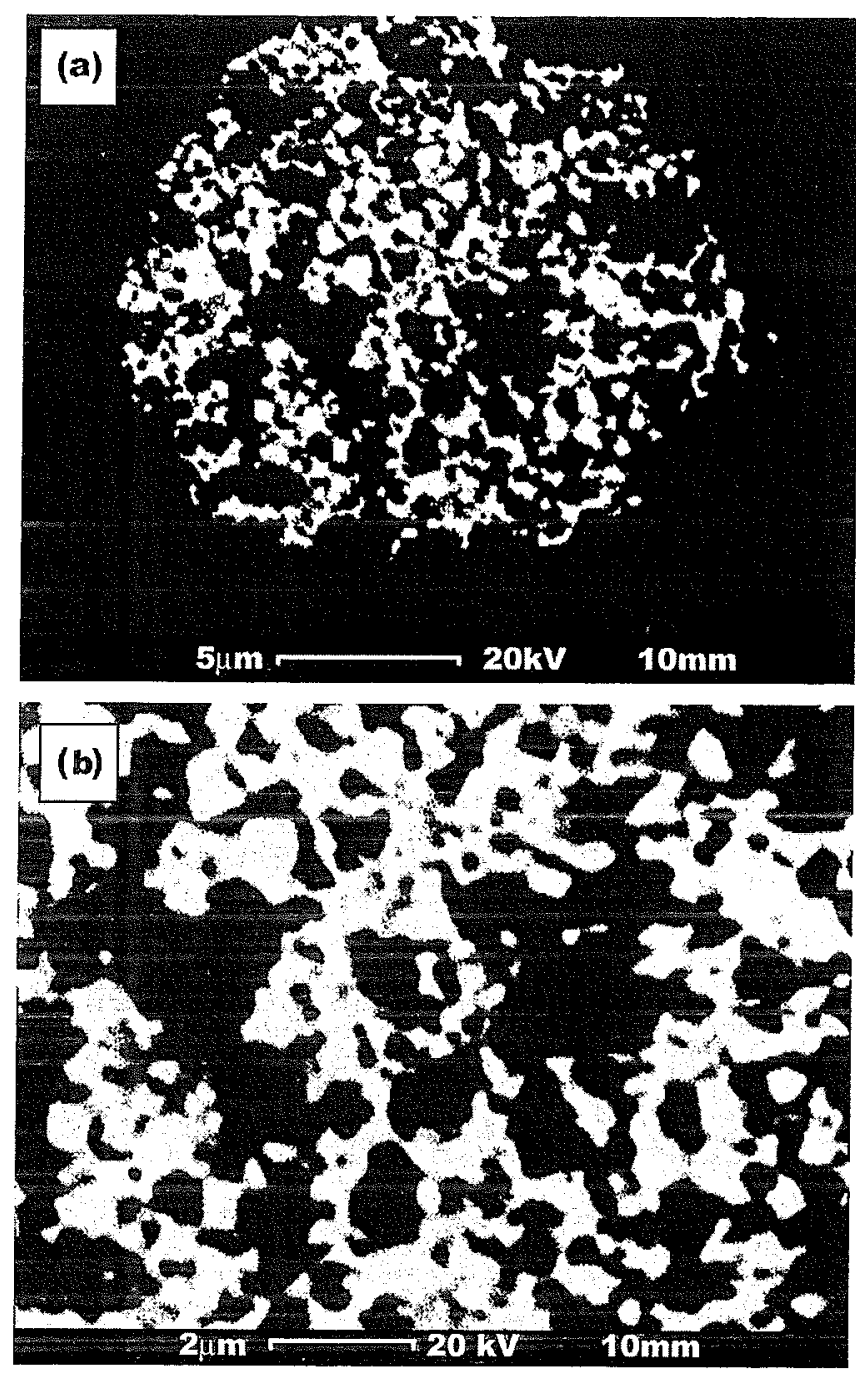

Fig. 6 (a) Overview and (b) detail distribution of (fc) WC (white) and $\mathrm{CoCr}$ (black)

In the first step, the raw materials were filled into an attritor together with a binder and a solvent. Due to the exact size control of the carbides and a homogenizing process without milling action, the carbide size distribution was very narrow.

After mixing, the suspension was spray-dried in a closedloop system under controlled atmosphere. The oxide content of the finished powder was between $100-300 \mathrm{ppm}$. Therefore it is comparable with gas atomized powders. The consequences of incompletely alloyed powders are an inhomogeneous corrosion resistance of the resulting coatings.

The produced micro pellets are very dense without any hollows or large pores (Fig. 5). The distribution of carbides and the metal matrix is also very homogenous (Fig. 6).

The following sintering step was done in a hydrogen-vacuum furnace. The first phase was the evaporation of the organic binder.

For the sintering process, different types of furnaces can be used. Generally temperatures are between 1100 and $1400^{\circ} \mathrm{C}$. The temperature and the sintering time influence the cohesion 
between the carbides and the metallic particles. Powders sintered at a too low temperature tend to burst or crack when they are injected in the nozzle or the burning chamber. Powders sintered at a too high temperature cannot be properly molten during the HVOF spraying. Internal porosity in the coating and low deposition efficiency are the consequence.

In spray-dried powders, the sintering causes small sinter bridges between the micro pellets. The destroying of these sinter bridges can be done with less energy. The grain sizes of the pellets are a result of the spray drying process and not of the crushing process. Therefore the use of heavy crushing equipment is not necessary. This powder was used for vacuum plasma spraying.

Table 1 Parameters for Slurry Abrasion Testing According to ASTM G 75

\begin{tabular}{ll}
\hline Parameters & \multicolumn{1}{c}{ Settings } \\
\hline Abrasive particles & $\mathrm{Al}_{2} \mathrm{O}_{3}$ \\
Particle size & $0.045-0.075 \mathrm{~mm}$ \\
Solid content in slurry & $50 \mathrm{wt} \%$ \\
Hardness of rubber matting & Shore $\mathrm{A} 67$ \\
Testing duration & $2 \mathrm{~h}$ up to $6 \mathrm{~h}$ in total \\
\hline
\end{tabular}

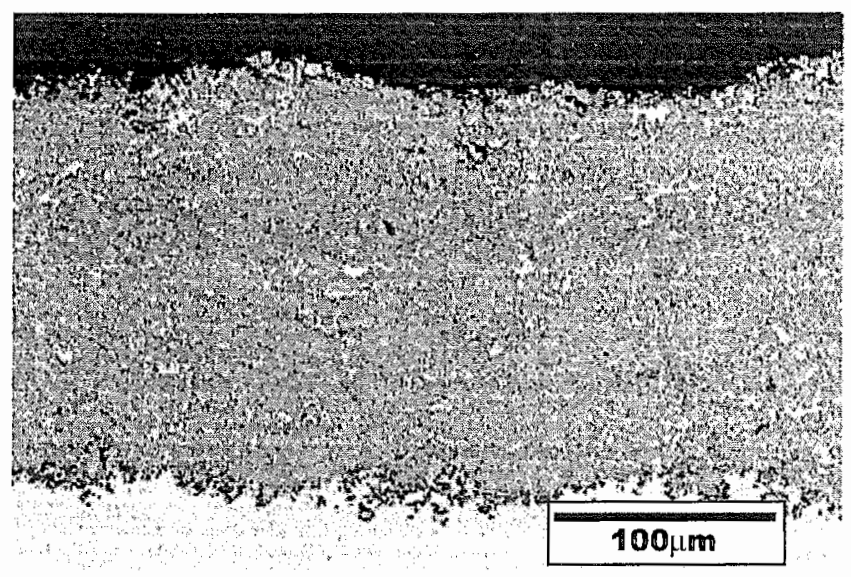

Fig. 7 Metallographically prepared cross section of conventional WC$\mathrm{CoCr}$ coating sprayed with a modified Terojet HVOF-system using kerosene
2.2.1 High Velocity Oxygen Fuel Spraying. For the HVOF tests, commercial equipment (Terojet), with some modifications working with kerosene and oxygen, was used. Kerosene-oxygen combustion is still the safest and simplest solution for the industrial design of HVOF systems. Because the particle acceleration is mainly determined by the particle size and the momentum flux of the jet, ${ }^{[11]}$ the system design has to coincide with the powder design. The final goal is controlling the heating of the spraying particles near the melting point of the metallic matrix and achieving maximum particle speed. High particle acceleration allows maximum particle speed in a given acceleration zone, as in the barrel itself. When the kinetic energy of a particle reaches the value larger than the bonding mechanism with the surface, the spray particle impacts following the rules of explosion plating. ${ }^{[12,13]}$

Figure 7 shows the metallographic cross section of a conventional HVOF sprayed WC-CoCr coating for later comparison with the nano-WC-CoCr vacuum plasma sprayed. The coating has no visible porosity and a very homogenous, chemically unchanged structure.

\section{Characterization}

In addition to the metallographical preparation, the coatings were examined using light-scanning, electron microscopy (SEM), and transmission electron microscopy (TEM). The chemical composition was controlled using $x$-ray diffraction (XRD), glow discharge optical emission spectroscopy (GDOES) ${ }^{[14-16]}$ energy dispersive $\mathrm{x}$-ray (EDX), and EELS.

Technological tests were performed to classify behavior to the different wear attacks.

\subsection{Wear Testing}

Wear testing was performed according to different standardized testing procedures. The following wear conditions were examined: abrasion and water jet erosion including erosive particles.

3.1.1 Abrasive Wear. The abrasive wear testing was done in accordance with ASTM G 75. ${ }^{[17]}$ This test is known as the Standard Test Method for Determination of Slurry Abrasivity (Miller Number) and Slurry Abrasion Response of Materials (SAR Number). The slurry abrasion test is a method used fre-

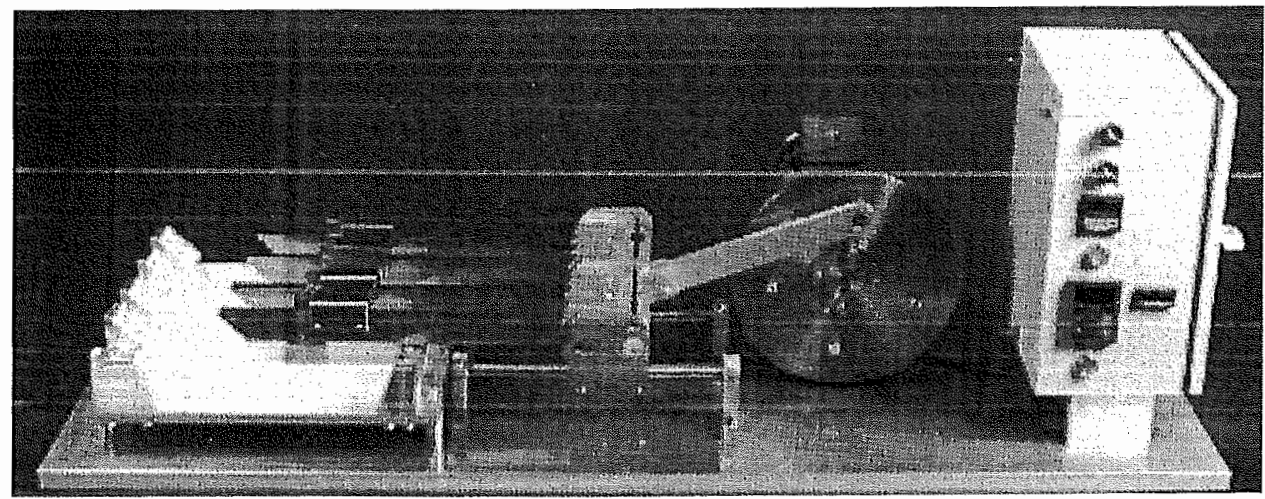

Fig. 8 Abrasion testing (4 samples at a time) according to ASTM G 75 Miller test 


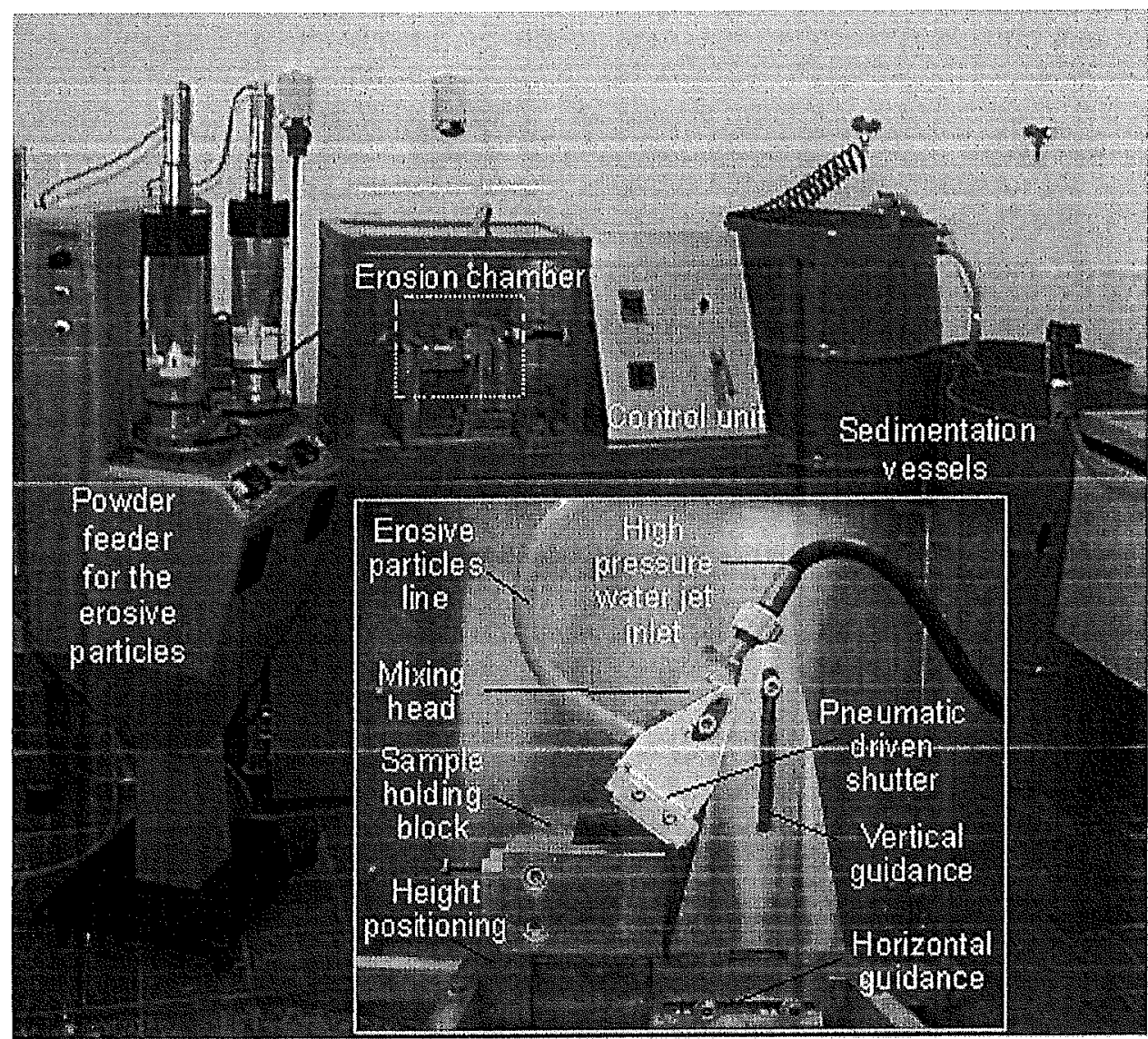

Fig. 9 Erosion testing using a high-pressure water jet with erosive particles

Table 2 Parameter for Water Jet Erosion Testing With Erosive Particles

\begin{tabular}{ll}
\hline Parameters & \multicolumn{1}{c}{ Settings } \\
\hline Solid erosive & $\mathrm{Al}_{2} \mathrm{O}_{3}$ \\
Particle size & $0.045-0.075 \mathrm{~mm}$ \\
Solid content & $0.03 \mathrm{wt} \%$ \\
Fluid velocity & $\mathrm{v}=160 \mathrm{~m} / \mathrm{s}$ \\
Impact angle & $30^{\circ}, 60^{\circ}, 90^{\circ}$ \\
\hline
\end{tabular}

quently to evaluate the wear rate of a solid surface exposed to hard particles moving along and forced against it (Fig. 8). The hard particles are mixed in a liquid so that the consistency is that of a slurry that could be pumped. The duration of the test is 3 times for $2 \mathrm{~h}$ each. Every $2 \mathrm{~h}$ the cumulative mass loss and the mass-loss-rate $(\mathrm{mg} / \mathrm{h})$ of the four equal samples are measured and the corresponding volume loss can be calculated. The testing conditions are summarized in Table 1.

3.1.2 Erosive Wear. The erosion testing was done in a combined test of ASTM G 73 (liquid impingement erosion testing) and ASTM G 76 (solid particle impingement). In this particular case, a water jet containing abrasive particles impinges on the surface to be tested. Impact angles between $15^{\circ}$ and $90^{\circ}$, standoff distance, content of abrasive material, and water jet velocity can vary. The testing device is shown in Fig. 9. The tests were carried out using the parameters given in Table 2 .

\section{Results}

\subsection{Low-Load Vickers Hardness}

The low-load Vickers hardness measurements (HV 0.3) of the vacuum plasma sprayed Fe17Cr10Mn6VMo coatings showed that the finer the starting powder was, the higher the coating hardness became (Fig. 10). A shorter spraying distance or a lower $\mathrm{Ar} / \mathrm{N}_{2}$ content led also to increased hardness values.

The fine WC-CoCr coatings showed slightly higher hardness values in case of the vacuum plasma sprayed coatings compared with the conventional HVOF sprayed ones (Table 3 ).

4.1.1 Abrasive Wear. The results of the abrasive Miller test (ASTM G75) for the Fe17Cr10Mn6VMo coatings are given in Fig. 11 for the $\mathrm{N}$ range from 0 to 3 wt. $\%$, where the maximum hardness was achieved. The abrasion resistance is comparable to hard plated $\mathrm{Cr}, \mathrm{T} 800$ or thermally sprayed $\mathrm{Al}_{2} \mathrm{O}_{3}$. The advantage is the lower hardness, which makes them easier to machine, but with the same corrosion resistance.

The abrasion results from the Miller test are shown in Fig. 12 for the WC-CoCr coatings in detail. For further comparison, a hard $\mathrm{Cr}$ coating was tested as well. The volume loss was calculated by using the density for hard-Cr $\left(\rho=7.19 \mathrm{~g} / \mathrm{cm}^{3}\right)$ and WC-CoCr $\left(\rho \approx 14.7 \mathrm{~g} / \mathrm{cm}^{3}\right)$. The abrasive wear resistance of the HVOF-sprayed WC-CoCr layer was a factor of 13 times more resistant than hard-Cr, and the vacuum plasma sprayed fc-WCCoCr coating was a factor of 9 . 


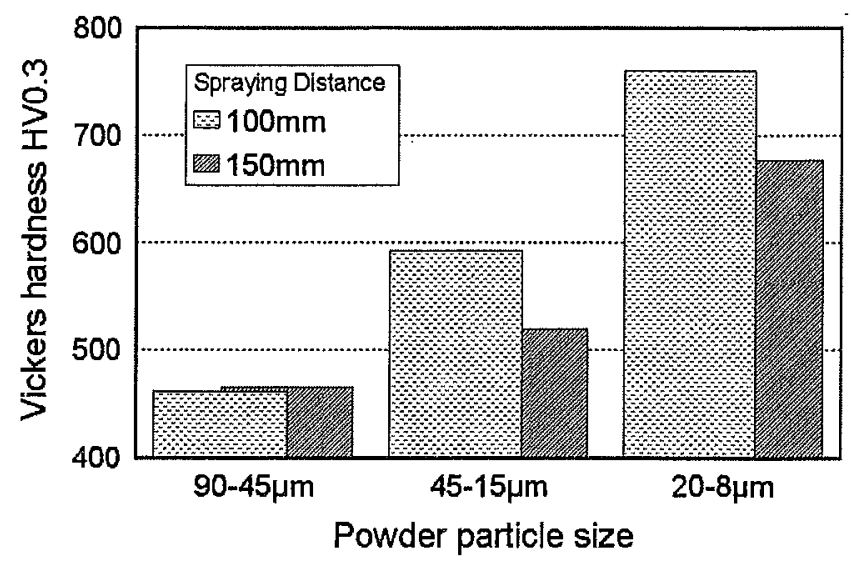

Fig. 10 Low-load Vickers hardness (HV 0.3) of reactively atmospheric plasma sprayed Fe17Cr10Mn6VMo coatings at different stand off distances and using different powder size distributions

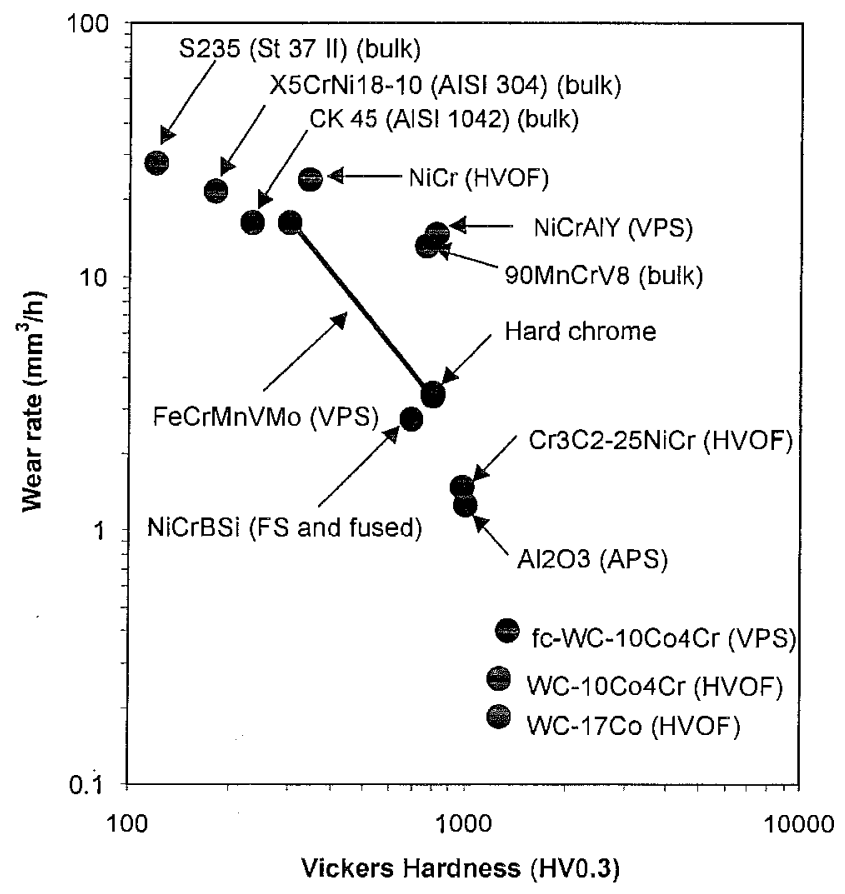

Fig. 11 Volume loss rate according to ASTM G75 test method of the investigated coatings and compared bulk materials as a function of their Vickers hardness (HV0.3)

Table 3 Low-Load Vickers Hardness (HV 0.3) of Thermally Sprayed WC-CoCr Coatings (Conventional WC by HVOF, Fine Carbides by VPS) Compared to, for Example, Hard Chromium

\begin{tabular}{lr} 
Material & HV 0.3 \\
\hline Hard Chromium & 750 \\
HVOF sprayed WC-CoCr & 1272 \\
VP sprayed (fc) WC-CoCr & 1345 \\
\hline
\end{tabular}

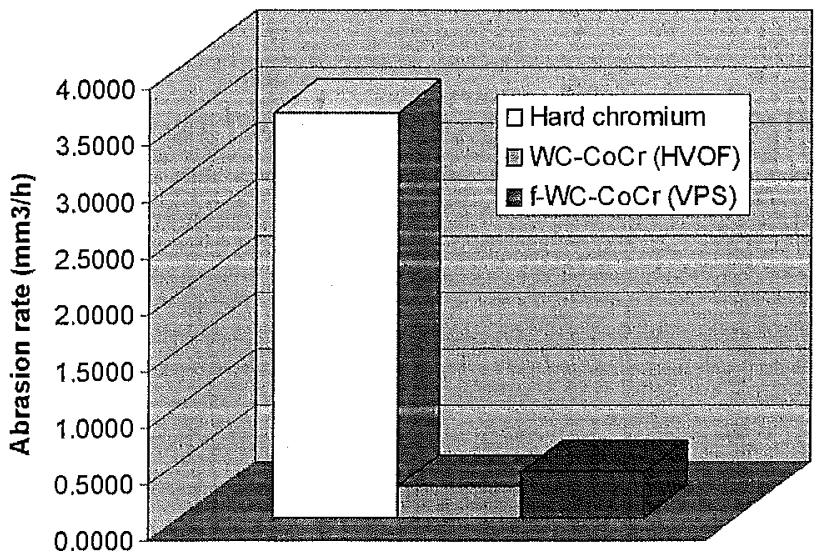

Fig. 12 Abrasion rates $\left(\mathrm{mm}^{3} / \mathrm{h}\right)$ measured by ASTM G75 Miller test of the HVOF sprayed conventional WC-CoCr coatings and VPS fc$\mathrm{WC}-\mathrm{CoCr}$ compared with hard $\mathrm{Cr}$

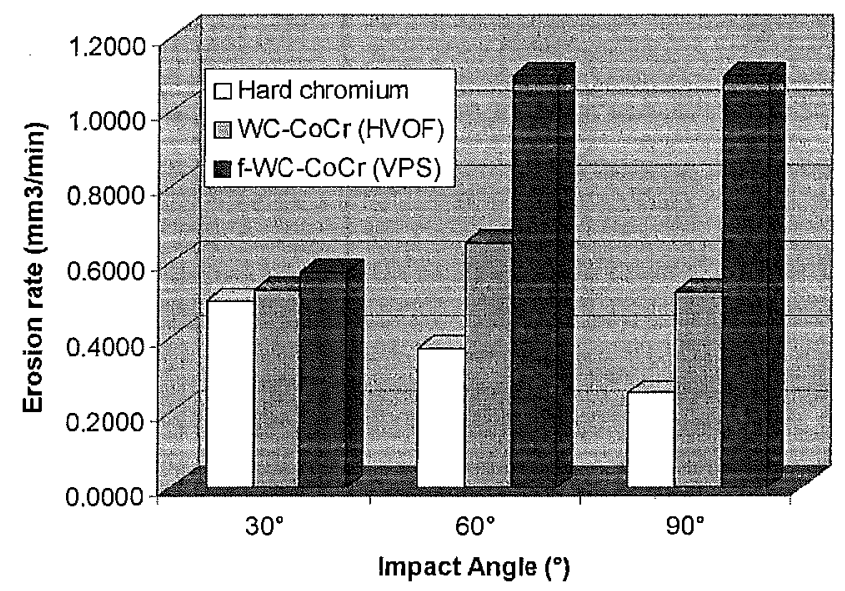

Fig. 13 Erosion rate $\left(\mathrm{mm}^{3} / \mathrm{min}\right)$ of fine WC-CoCr vacuum plasma and conventional HVOF sprayed coatings at different water jet impact angles

4.1.2 Erosive Wear. The erosion resistance, especially in case of perpendicular impact, gives information on the toughness of a layer. The results of all the measurements are compared with hard-Cr for impact angles of $30^{\circ}, 60^{\circ}$, and $90^{\circ}$ (Fig. 13).

The WC-CoCr coatings made by HVOF spraying do not show a clear dependence on the impact angle, whereas the VPS coatings show a clear increase in wear with increasing angle, contrary to the hard $\mathrm{Cr}$ layers. This effect can be explained by the higher hardness and brittleness of the layers (especially at high impact angles) due to the higher temperatures during VPS processing.

\section{Summary and Conclusions}

The possibility of using the VPS-process for reactive spraying of Fe-based alloys to improve the wear resistance has been proved within this study. It has been shown that an increase in energy of the plasma yields an increase in $\mathrm{N}$ content of the coating. The use of an additional $\mathrm{N}_{2}$-rich environment at the 
substrate could yield a further increase in $\mathrm{N}$ content of the coating. The basic advantage of these nano-MMC Fe-based alloys is:

- lower price compared with Ni- or Co-based alloys,

- easier machineability than conventional cermets, and

- higher spray rate and deposition efficiency than usual ceramics.

The vacuum plasma sprayed submicron WC-CoCr coatings exhibited a higher hardness but a lower abrasion and erosion resistance compared with the conventional WC-CoCr HVOF sprayed coatings.

\section{Acknowledgments}

Mr. F. Fischer from WOKA Schweißtechnik GmbH (Barchfeld, Germany) is acknowledged for the preparation and delivery of the WC-CoCr powders used for HVOF and vacuum plasma spraying. The project "Thermally Sprayed Protection Coatings with Highest Resistance Against Wear and Corrosion Made by Nanostructured Powders" was partially financial supported by the Swiss Priority Program on Materials Research (PPM). The Institute of Metallurgy of the Swiss Federal Institute of Technology is acknowledged for the alloy design and the TEM investigations.

\section{References}

1. N. Wang, Z. Wang, K.T. Aust, and U. Erb: "Effect of Grain Size on Mechanical Properties of Nanocrystalline Materials," Acta Metall. Mater., 1995, 43, pp. 519-28.

2. T.D. Xiao, S. Jiang, C.W. Strock, P.R. Strutt, D.M. Wang, Y. Wang, and R. Zatorski: "Thermal Spray of Nanostructured Ceramic Coatings for Improved Mechanical Properties" in Proceedings of 12th International Conference on Surface Modification Technologies, ASM International, Materials Park, OH, 1998, pp. 489-93.

3. K.A. Padmanabhan: "Mechanical Properties of Nanostructured Materials," Mater. Sci. Eng. A, 2001, 304-306, pp. 200-05.

4. H. Gleiter: "Nanostructured Materials: Basic Concepts and Microstructure," Acta Mater., 2000, 48, pp. 1-29.

5. O. Brandt and S. Siegmann: "Eigenschaftsprofil Nanostrukturierter Schichten, Hergestellt Durch das Thermische Spritzen," Verbundwerkstoffe und Werkstoffverbunde, Wiley-VCH Verlag GmbH, Weinheim, Germany, 1999, pp 664-70 (in German)

6. O.C. Brandt and St. Siegmann: "Possibilities of Reactive Thermal
Spraying of New Ferrous Alloys (D)" in Proceedings of 2nd United Thermal Spray Conference, DVS Verlag, Düsseldorf, Germany, $l$, 1999, pp. 259-64.

7. O. Brandt and St.D. Siegmann: "VPS Coatings Using Nanostructural Iron-Based Alloys" in Proceedings of 15 th International Thermal Spray Conference-Thermal Spray: Meeting the Challenges of the 21st Century, ASM International, Materials Park, OH, 2, 1998, pp. 1249-53.

8. S. Siegmann and O. Brandt: "Thermally Sprayed Protection Coatings with Highest Resistance Against Wear and Corrosion, Made by Nanostructured Powders," PPM - Swiss Priority Program on Materials Research Final Report 1995-99, D. Landolt, and D. Reinhard, eds., EPFL Lausanne, Lausanne, 2000, pp. 201-16.

9. W-F. Bähre, C. Solenthaler, P.J. Uggowitzer, and M.O. Speidel: "Hardenable Austenitic Iron Base Metal-Matrix-Composites" in Proceedings of PM Tech '97, 1997, p. 12.

10. F.F. Fischer, M.D. Dvorak, and St. Siegmann: "Development of Ultra Thin Carbide Coatings for Wear and Corrosion Resistance" in Proceed ings of Thermal Spray 2001-New Surfaces for a New Millennium, C.C. Berndt, K.A. Khor, and E.G. Lugscheider, ed., ASM International, Materials Park, OH, 2001, pp. 1131-35.

11. H.J. Richter and H.-D. Schumacher: "Thermodynamische und Strömungstechnische Grundlagen des HochgeschwindigkeitsFlammspritzens" in Proceedings of 3. Kolloquium-Hochgeschwindigkeits-Flammspritzen, Gemeinschaft Thermisches Spritzen e.V (GTS), c/o Linde AG, ed., Höllriegelskreuth, 1994, pp. 15-19 (in German).

12. M. Dvorak and J.A. Browning: "Extending the Limits of HVOF" in Proceedings of 14th International Thermal Spray Conference: Thermal Spraying-Current Status and Future Trends A. Ohmari, ed., High Temperature Society of Japan, 1, 1995, pp. 405-09.

13. M. Dvorak and R. Torday: "Eigenschaften von mit hohem Brennkammerdruck HVOF-gespritzten Schichten" in Proceedings of Thermische Spritzkonferenz TS 96 175, E. Lugscheider, ed., Deutscher Verlag für Schweisstechnik DVS-Verlag, Düsseldorf, Germany, 1996, pp. 6-9 (in German).

14. St. Siegmann: "Schichtcharakterisierung mittels Glimmentladungsspektroskopie (GDOS)" in Proceedings of Tribologie Forum, St. Sicgman, ed., EMPA Thun, 1995, pp. 14-22, (in German).

15. D. Delfosse and M. Aeberhard: Tiefenprofilanalysen von technischen Schichten und Oberflächen-Teil 1: Grundlagen der GDOS und Beispiele aus der Galvanik, Oberflächen - Polysurfaces, 1997, pp. 7-10, (in German).

16. D. Delfosse, M. Aeberhard, and S. Sgobba: Tiefenprofilanalysen von technischen Schichten und Oberflächen-Teil 2: Diffusionsstudien und Analysen von nichtleitenden Beschichtungen mit der GDOS, Oberflächen-Polysurfaces (1998), pp. 24-28, (in German)

17. Anon., ASTM Designation G 75-95: "Test Method for Determination of Sluny Abrasivity (Miller Number) and Slurry Abrasion Response of Materials (SAR Number)" in Annual Book of ASTM Standards, ASTM, Philadelphia, PA, 1995. 